\title{
RETROSPECTIVE STUDY OF PERIODONTAL PATIENTS PROFILE IN A TEACHING INSTITUTION
}

T.B. Taiyeb Ali, I.A. Razak. Retrospective study of periodontal patients profile in a teaching institution. Annal Dent Univ Malaya 2000; 7: 22-26.

\section{ABSTRACT}

The purpose of the present study was to determine the periodontal profile, toothbrushing habits and oral hygiene status of patients referred to a teaching institution for periodontal treatment, based on patients' treatment records. A total of 207 consecutive patients diagnosed with periodontitis who had been interviewed and clinically examined over a period of approximately one year were included in this study. The assessments of plaque levels, bleeding on probing (BOP) scores, probing pocket depth (PPD) and degree of bone loss from radiographs were made. The average age of this study group was $45 \pm 12.8$ years old with an age range of 20 to 76 years. The subjects comprised of $40 \%$ Malays, 34\% Chinese and 26\% Indians. The male to female ratio was almost $1.5: 1$. These patients presented with high plaque and BOP scores. These findings do not concur with the high frequency of toothbrushing reported $(86.5 \%$ of subjects brushing twice or more times per day). This may reflect on the ineffectiveness of brushing as evident from their high plaque scores. Periodontal pockets were detected in $69 \%$ of the teeth in these patients with an average of 17 teeth per patient being involved. Probing pocket depths of $>6 \mathrm{~mm}$ was found on the average in 3 teeth per patient. Alveolar bone loss as assessed from radiographs was detected in $52 \%$ of the teeth in this study group of which 12 teeth per patient on the average exhibited this. Advanced bone loss involving about $2 / 3$ and more of the root length was detected on the average on 1 tooth per patient. Early onset periodontitis accounted for about $5 \%$ of the total cases seen. About $21 \%$ of subjects suffered from advanced adult periodontitis. Hence about a-quarter of the patients referred already had marked periodontal destruction.

Key words: periodontitis, periodontal status, plaque, bleeding on probing, bone loss, probing pocket depth.

\section{INTRODUCTION}

The human adult population at large is susceptible to periodontal breakdown in varying degrees of severity (1). Although microbial plaque is the main etiological factor (2), the host's defense mechanisms against this microbial insult has also a major role in determining the ultimate extent of destruction of the periodontium (3-5). The severity of periodontal disease is also affected by social factors such as age, sex, race, occupation, socio-economic status, education and whether they live

\section{Original Article}

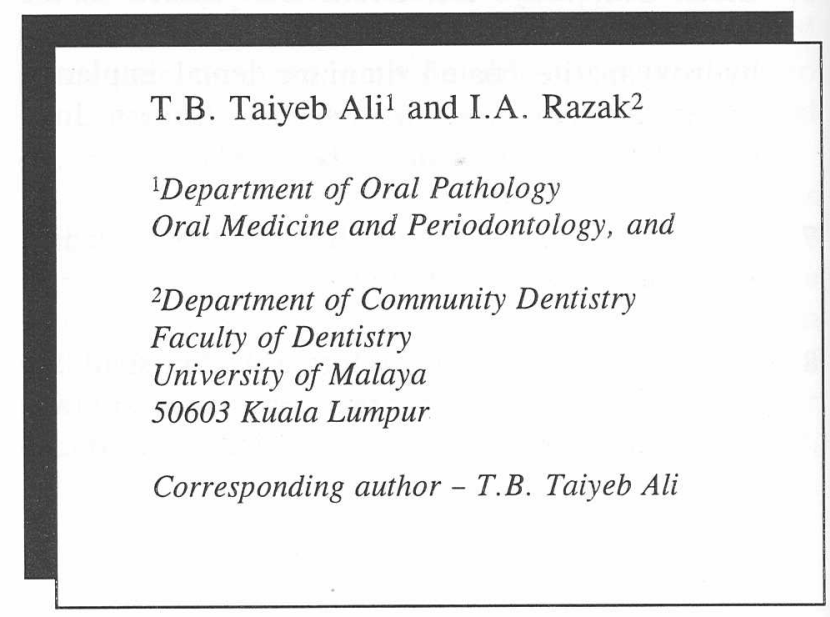

in urban or rural areas (6). The spectrum is further narrowed with emphasis placed particularly on the susceptible individuals. In a recent review by Clark \& Hirsh (1995) (7), the various risk factors implicated that come into play may add up to increase the susceptibility in a person. There is also ample evidence that there is some genetic basis for some forms of periodontal diseases particularly the early onset types, which are manifested, in the young individuals. Many facets of the immune responses are under genetic control.

Prevalence of periodontal disease and its severity in different populations has been extensively studied (89). In Malaysia, as in many other developing nations, periodontal disease is a problem of worrying magnitude $(10-11)$. However there is a dearth of data not only on the periodontal status in adults, the distribution of the different types of periodontal diseases or the association with risk factors. In the first of surveys done in this country, which was part of the Interdepartmental Committee on Nutrition for National Defence (ICNND) nutrition survey in 1954 (12), Russell's Periodontal Index was used. It was found that the 15 to 19 yearsold scored 0.17 and those 50 years and above had a score of 4.48. In the epidemiological survey of 9,047 adults in Malaysia reported in 1977 (10), $72 \%$ had periodontal disease ranging from mild inflammation, intense gingivitis and destructive periodontal disease. Mild stage was exhibited in $31 \%$, while $20 \%$ had intense gingivitis. The average number of teeth with pockets greater than $3 \mathrm{~mm}$ was $1.2 \pm 2.8$ per patient. The percentage of Indian/Pakistani with intense gingivitis was comparatively low but the prevalence of destructive stage was high. It was assumed that this ethnic group was more prone to destructive periodontitis or the disease progresses more rapidly in this ethnic group. This was reflected in the greater tendency in these individuals to possess teeth with pockets greater than 3 $\mathrm{mm}$. About $81 \%$ of the subjects brushed their teeth with no appreciable difference between the 2 genders. About $13 \%$ did not have any cleansing habit with about $5 \%$ 
utilizing other forms of cleansing which included chewing twigs or fingers with powdered charcoal or salt.

The above trends in disease prevalence were again reflected in the recent dental epidemiological survey of 13,740 adults in Malaysia by the Ministry of Health (1993) (11). Periodontal health was assessed using the CPITN (Community Periodontal Index of Treatment Needs). Only about $7 \%$ of the subjects examined had no periodontal disease. Females $(8 \%)$ had healthier periodontium compared to males $(6 \%)$. The urban population $(10 \%)$ had no disease, had better periodontal status than those in rural areas $(6 \%)$. Periodontal pockets of $4-5 \mathrm{~mm}$ were detected in $17 \%$ of the subjects; with $6 \%$ of the subjects exhibiting deep periodontal pockets of $6 \mathrm{~mm}$ and more. Complex periodontal treatment was required by less than $15 \%$ of the sample.

Using the CPITN, Rahimah (13) assessed the periodontal conditions of 779 subjects in selected occupational adult groups, aged between 20-54 years. These groups comprised navy personnel, government workers, factory workers, villagers and rubber tappers. Only $16 \%$ of the adults had healthy periodontium and this was mainly in the navy personnel and factory workers. Bleeding was limited to the 20-24 years-old group and calculus was highly prevalent in about $65.5 \%$ of the subjects. Mostly shallow pockets were detected. Deep pockets with prevalence ranging from $8-25 \%$ were detected only in the rubber tappers in the older ages category.

In a study by Esa et al (14) on oral hygiene practices among Malaysian adult it was reported that about $90 \%$ of the subjects brushed their teeth, of which $57 \%$ brushed twice a day. The remaining $8 \%$ brushed and flossed while $2 \%$ used other methods of tooth cleaning.

The low priority for dental health and possibly the lack of dental consciousness and knowledge among the general population could possibly contribute to the apathy in the periodontal health in the Malaysian adults. In addition the increase in the retention of the dentition due to the reduction in tooth loss from dental caries as a result of the nationwide fluoridation programme for the past years and the widespread use of fluoridated toothpaste in this country, may have resulted in a greater number of teeth being exposed to the ravages of periodontal disease.

The purpose of the present study is to describe the periodontal profile of the patients referred to the Periodontal Unit of Faculty of Dentistry, University of Malaya, for periodontal treatment in terms of dental cleansing, oral hygiene and severity of periodontal destruction.

\section{MATERIAL AND METHODS}

The records of 207 consecutive patients referred to the periodontal unit and who had been diagnosed as having periodontitis were included in this study. The patients had been interviewed and their name, age, sex, ethnicity, medical health status, dental cleansing and smoking habits had been recorded. The patients had been examined clinically and radiographically. The plaque (PS) and bleeding on probing (BOP) scores had been charted as either absent or present on 4 surfaces of each tooth and the percentage of plaque and BOP scores were calculated. The probing pocket depths (PPD) had been measured using William's probe on 6 surfaces of the teeth. The highest reading on each tooth was recorded. Rotational tomogram (OPG) radiographs had been taken. The greatest amount of bone loss in relation to the thirds of the root length for each tooth was noted.

\section{RESULTS}

The age range of these 207 patients was 20-76 years with the average age $( \pm S D)$ of this group being 45 $( \pm 12.8)$. The male:female ratio was almost $1.5: 1$. The distribution of the subjects according to ethnic grouping is given in Fig. 1. Malays comprised $40 \%$ of the patients, Chinese $34 \%$ and Indians $26 \%$. These patients were all from urban areas.

The toothbrushing frequency of this group of patients is shown in Table 1, with $86.5 \%$ brushing regularly twice or more times per day. There was however, only a very small percentage of $1.4 \%$ who brushed infrequently.

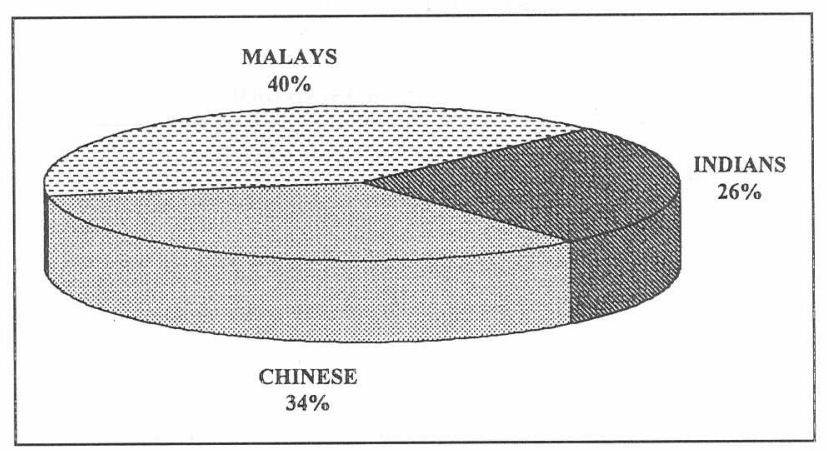

Figure 1: Ethnicity of the 207 patients.

Table 1. Frequency of toothbrushing per day in the Referred 207 patients

\begin{tabular}{lcc}
\hline Number of times/day & No. of response & \% response \\
\hline Do not brush daily & 3 & 1.4 \\
Once per day & 25 & 12.1 \\
Twice per day & 149 & 72.0 \\
$\begin{array}{lc}\text { More than two times } \\
\text { per day }\end{array}$ & 30 & 14.5 \\
\hline \multicolumn{1}{l}{ Total } & 207 & 100.0 \\
\hline
\end{tabular}




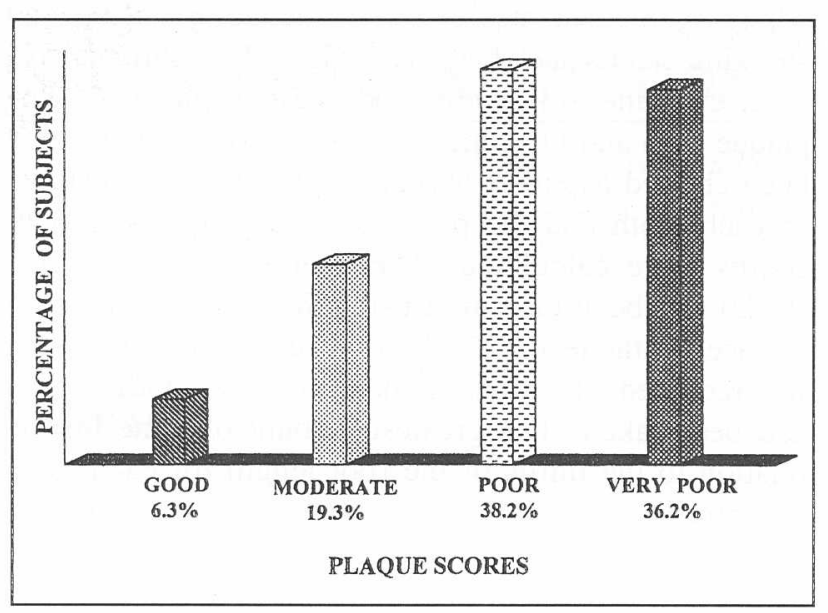

Figure 2: Distribution of plaque scores in the 207 patients.

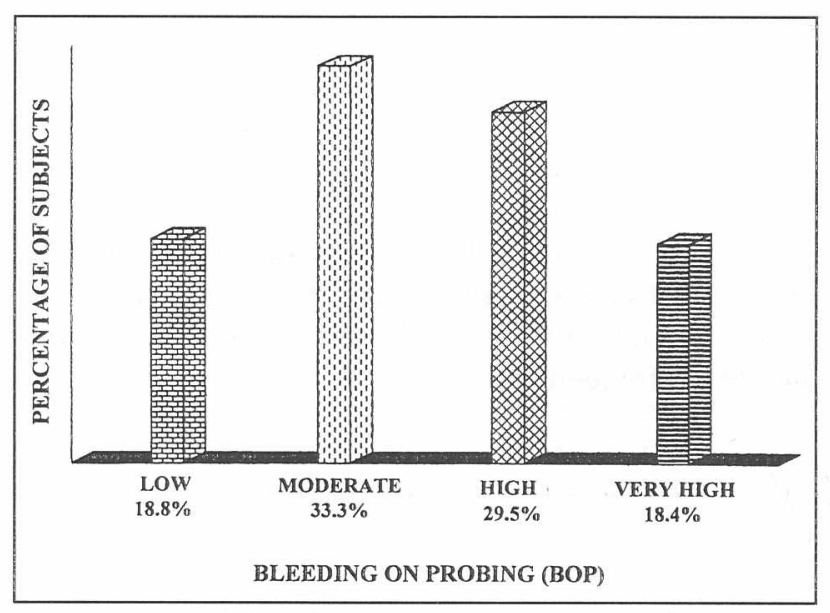

Figure 3: Distribution of bleeding on probing (BOP) scores in the 207 patients.

The plaque scores of the patients were categorized into good (0-25\%), moderate (26-50\%), and poor (51$75 \%)$ and very poor (76-100\%) and its distribution in the patients are shown in Fig. 2. Most of the patients had poor to very poor plaque scores categories.

The BOP scores calculated in percentage scores were similarly categorized in 4 groups namely, low (0$25 \%)$, moderate $(26-50 \%)$, high $(51-75 \%)$ and very high $(76-100 \%$ ) (Fig. 3). About $33 \%$ and $29 \%$ of the patients had moderate and high bleeding scores respectively.

In this selected population group the average number of teeth with pockets was found to be 17 teeth per patient, $69 \%$ of the teeth had pockets. The pockets were divided into 3 groups, namely shallow (3-4 mm), moderate $(5-6 \mathrm{~mm})$ and deep $(>6 \mathrm{~mm})$ and its distribution in these patients is given in Table 2. Probing pocket depths of $>6 \mathrm{~mm}$ was found on the average in 3 teeth per patient.

The average number of teeth with bone loss was 12 teeth per patient with $52 \%$ of the teeth showing bone loss in these 207 patients. The extent of bone loss was
Table 2. Total number of teeth with pockets and the mean number of pockets per patient

\begin{tabular}{lcr}
\hline Pocket Depth & Total $(\%)$ & Mean \pm SD \\
\hline Shallow & $1527(44.4 \%)$ & $7.4 \pm 5.8$ \\
Moderate & $1261(36.7 \%)$ & $6.1 \pm 4.4$ \\
Deep & $648(18.7 \%)$ & $3.1 \pm 4.4$ \\
$\begin{array}{l}\text { Total number of teeth } \\
\text { with pockets }\end{array}$ & $3436(100 \%)$ & $16.6 \pm 8.3$ \\
\hline * Total number of teeth examined & $=4965$ \\
Percentage of teeth with pockets & $=\frac{3436}{4965} \times 100 \%=69.2 \%$
\end{tabular}

Table 3. Total number of teeth with bone loss and the mean number of teeth with boss loss per patient

\begin{tabular}{lrr}
\hline Extent of bone loss & Total (\%) & Mean \pm SD \\
\hline Early & $1376(53.6 \%)$ & $6.7 \pm 5.9$ \\
Moderate & $891(34.7 \%)$ & $4.3 \pm 4.2$ \\
Advanced & $302(11.7 \%)$ & $1.5 \pm 2.3$ \\
$\begin{array}{l}\text { Total number of teeth } \\
\text { with bone loss }\end{array}$ & $2572(100 \%)$ & $12.4 \pm 5.1$ \\
\hline * Total number of teeth examined & $=4965$ \\
Percentage of teeth with bone loss & $=\frac{2572}{4965} \times 100 \%=51.8 \%$ \\
&
\end{tabular}

Table 4. The types of periodontitis diagnosed in the 207 patients

\begin{tabular}{lc}
\hline Diagnosis & Percentage \\
\hline Early Adult Periodontitis & 33.8 \\
Moderate Adult Periodontitis & 40.6 \\
Advanced Periodontitis & 20.8 \\
Rapidly Progressive Periodontitis & 2.9 \\
Juvenile Periodontitis & 1.9 \\
\hline
\end{tabular}

categorized into early (until 1/3 root length), moderate $(1 / 3-2 / 3$ root length) and advanced $(>2 / 3$ root length). Its total and mean distribution in these 207 subjects is presented in Table 3 . On the average advanced bone loss was noted on less than 2 teeth per patient.

The diagnosis of the different types of periodontitis is shown in Table $4.33 .8 \%$ of this group was diagnosed as having early periodontitis, $40.6 \%$ moderate and $20.8 \%$ advanced periodontitis. About $5 \%$ of these patients were diagnosed with Early Onset Periodontitis. 


\section{DISCUSSION}

Periodontal disease was found to be widespread in the Malaysian population in the two large epidemiological surveys carried out by the Ministry of Health (10-11). Hence, the present study was aimed at determining the profile of patients who had been referred for periodontal treatment to the Faculty of Dentistry, University of Malaya, which forms one of the few centres for referral of these cases. The purpose is to add to the meagre pool of data particularly on the severity of periodontal diseases in the country since every tooth in the mouth was assessed in this investigation.

It is interesting to note that the number of male patients were greater in this study, possibly indicating comparatively more disease in this gender than in the females, as documented in various studies (15-17). In reference to the different ethnic groups, the greater percentage of Indians in this study population in relation to their proportion in the general population possibly reflects the greater susceptibility to destructive periodontal breakdown in the adults of this ethnic group. This trend was also noted in both the national epidemiological studies. Could this be related to some inherent susceptibility or is it just a matter of poorer plaque control or possibly a different bacterial spectrum in this ethnic group probably needs further investigation.

Addressing behavioral factors like oral hygiene and frequency of brushing in this investigation, the majority of the patients brushed their teeth twice or more per day, which was also reported in the general population by Esa et al (14). It is somewhat surprising that inspite of the good practice of twice daily brushing; the oral hygiene status as reflected by the plaque scores displayed poor status, indicating ineffective dental cleansing. The high BOP scores, which serve as a presumptive indicator for disease activity (18), with $69 \%$ of the teeth exhibiting pocketing and $52 \%$ of the teeth showing bone loss respectively commensurate with the poor oral hygiene.

The fact that about $70 \%$ of the dentition exhibited pocketing and on the average each patient had deep pockets on 3 teeth (Table 2 ) indicate a fair amount of periodontal breakdown. This however contradicts the findings by Rahimah (13), who detected deep pockets only in the rubber tappers, who probably live in rural areas. Here the patients covered the full spectrum of occupational groups and comprised of an urban population but a direct comparison is not possible, as these are referred patients. It could only implicate that the urban and probably better educated population do exhibit fairly marked periodontal breakdown. Either apathy or lack of knowledge on the part of the patient may possibly explain the findings in this investigation or the dentist overlooked the periodontal disease until disease progressed to manifest disturbing signs and symptoms. Oral health education campaigns have to highlight periodontal care and periodontal diseases along with its consequences namely tooth mobility and ultimate tooth loss to the general public. In addition our sophisticated dental delivery system is in need of the services of dental auxiliaries, particularly the dental hygienist to deliver prophylactic procedures, oral health education and supportive periodontal therapy to the adult population. During the organization of these services, there is also a need to consider other general factors so as to optimize benefits of the services rendered.

In this study, early-onset periodontitis (EOP) was noted in $4.8 \%$ of these patients. At this juncture, it could be suggested that the screening of young individuals or young adults in the general dental practice and government clinics be initiated so that this form of periodontal disease can be detected early and treatment instituted before the advanced stage or loss of teeth results.

Finally, it is to be reminded that the results of this study should be interpreted with caution as it is a biased population sample pertaining to only patients referred for periodontal problems and having been diagnosed with periodontitis. Hence they possibly constitute the risk group and probably those susceptible to periodontal breakdown.

In conclusion, this study demonstrated that the majority of the patients referred to the Faculty of Dentistry, University of Malaya for periodontal treatment had fairly severe periodontal breakdown and poor oral hygiene status. It was found that about onequarter of the patients referred already had marked periodontal destruction.

\section{REFERENCES}

1. WHO. Epidemiology, etiology and prevention of periodontal diseases. Geneva: Technical Report Series. 621, 1978.

2. Loe H, Theilade E, Jensen S. Experimental Gingivitis in man. J Periodontal 1965; 36: 177-187.

3. Theilade E. The non-specific theory in microbial etiology of inflammatory diseases. J Clin Periodontol 1986; 13: 905-911.

4. Slots J. Bacterial specificity in adult periodontitis. A summary of recent work. J Clin Periodontol $1986 ; 13: 912-917$.

5. Seymour GJ. Importance of the host response in the periodontium. J Clin Periodontol 1991; 18: 421426.

6. Wilton, JMA. Unchanging, subject-based risk factors for destructive periodontitis: race, sex, genetic, congenital and childhood systemic diseases. In: Johnson N.W. (ed): Markers of disease susceptibility and activity for periodontal diseases. Cambridge University Press. Cambridge. 1991 p 109-138. 
7. Clark NG, Hirsch RS. Personal risk factors for generalized periodontitis. J Clin Periodontol 1995; 22: $136-145$.

8. Pilot T, Miyazaki H. Global Results: 15 years of CPITN epidemiology. Int. Dent J 1944; 44: 553560.

9. Pilot $\mathrm{T} \&$ Barnes DE. An update on periodontal conditions in adults, measured by CPITN. Int. Dent J 1987; 37: 169-172.

10. Dental Division, Ministry of Health, Malaysia. Dental Epidemiological Survey of Adults in Peninsular Malaysia, Sept. 1974 - April 1975. National Printers Malaysia 1978.

11. Dental Services Division, Ministry of Health, Malaysia. Dental Epidemiological Survey of Adults in Malaysia 1990. National printers Malaysia 1993.

12. Interdepartmental Committee on Nutrition for National Defence (ICNND). Federation of Malaya Nutrition Survey. Washington DC: US Government Printing Service, 1962.
13. Rahimah AK. Profile of periodontal conditions in selected West Malaysian adults. Singapore Dent J 1994; 19: 4-7.

14. Esa R, Razak IA, Raja Latifah RJ, Jaafar N. A Survey on Oral Hygiene Practices among Malaysian adults. Clin. Prev. Den. 1992; 14: 23-27.

15. Hansen DF, Bjertness E, Gronnesby JK. A socioecologic model for periodontal diseases. J Clin Periodontol 1993; 20: 584-590.

16. Douglas CW, Gillings D, Sollecite W, Gammon M National trends in the prevalence and severity of the periodontal diseases. J Amer Dent Assoc 1983; 107: 403-412.

17. Tervonen T, Knuuttila M, Nieminen P. Risk factors associated with abundant dental caries and periodontal pocketing. Community Dent and Oral Epidemiol. 1991; 19: 82-87.

18. Lang NP, Joss A, Orsanic T, Gusberti FA, Siegrist BE. Bleeding on probing - A predictor for the progression of periodontal disease. J Clin Periodontol 1986; 13: 590-596. 